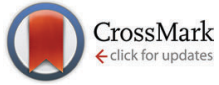

Cite this: J. Mater. Chem. C, 2015, 3, 10525 Accepted 31st July 2015

DOI: $10.1039 / \mathrm{c} 5 \mathrm{tc0} 01509 \mathrm{j}$

www.rsc.org/MaterialsC
Received 26th May 2015,

\section{Heterostructures of skutterudites and germanium antimony tellurides - structure analysis and thermoelectric properties of bulk samples $\dagger$}

\author{
Felix Fahrnbauer, ${ }^{a}$ Stefan Maier, $\ddagger^{\mathrm{b}}$ Martin Grundei, ${ }^{\mathrm{b}}$ Nadja Giesbrecht, \\ Markus Nentwig, ${ }^{\text {ab }}$ Tobias Rosenthal, ${ }^{b}$ Gerald Wagner, ${ }^{a}$ G. Jeffrey Snyder ${ }^{c d}$ and \\ Oliver Oeckler*a
}

\begin{abstract}
Heterostructures of germanium antimony tellurides with skutterudite-type precipitates are promising thermoelectric materials due to low thermal conductivity and multiple ways of tuning their electronic transport properties. Materials with the nominal composition [CoSb $\left.2(\mathrm{GeTe})_{0.5}\right]_{x}(\mathrm{GeTe})_{10.5} \mathrm{Sb}_{2} \mathrm{Te}_{3}(x=0-2)$ contain nano- to microscale precipitates of skutterudite-type phases which are homogeneously distributed. Powder X-ray diffraction reveals that phase transitions of the germanium antimony telluride matrix depend on its GeTe content. These are typical for this class of materials; however, the phase transition temperatures are influenced by heterostructuring in a beneficial way, yielding a larger existence range of the intrinsically nanostructured pseudocubic structure of the matrix. Using microfocused synchrotron radiation in combination with crystallite pre-selection by means of electron microscopy, single crystals of the matrix as well as of the precipitates were examined. They show nano-domain twinning of the telluride matrix and a pronounced structure distortion in the precipitates caused by GeTe substitution. Thermoelectric figures of merit of $1.4 \pm 0.3$ at $450{ }^{\circ} \mathrm{C}$ are observed. In certain temperature ranges, heterostructuring involves an improvement of up to $30 \%$ compared to the homogeneous material.
\end{abstract}

\section{Introduction}

In the search for novel thermoelectric materials for application in waste-heat recovery, micro- and nanostructured composites have become a major field of research. In addition to doping experiments that aim at adjusting an optimal charge carrier concentration, domain boundaries efficiently reduce the phononic part $\kappa_{\mathrm{ph}}$ of the thermal conductivity $\kappa \cdot .^{1-3}$ This is favorable in terms of a high thermoelectric figure of merit $Z T=\sigma S^{2} T \kappa^{-1}(\sigma=$ electrical conductivity, $S$ = Seebeck coefficient). While interfaces may

\footnotetext{
${ }^{a}$ Institute for Mineralogy, Crystallography and Materials Science,

Leipzig University, Scharnhorststr. 20, 04275 Leipzig, Germany.

E-mail: oliver.oeckler@gmx.de

${ }^{b}$ Department of Chemistry, Ludwig Maximilian University, Butenandtstr. 5-13, 81377 Munich, Germany

${ }^{c}$ Materials Science, California Institute of Technology, 1200 E. California Blvd., Pasadena, 91125 CA, USA

${ }^{d}$ Department of Materials Science and Engineering, Northwestern University, 2220 Campus Drive, Evanston, IL 60208-3109, USA

$\dagger$ Electronic supplementary information (ESI) available: Detailed information on syntheses and analyses, further Rietveld refinements and electron microscopy, additional information on the structure refinement from single-crystal data and thermoelectric properties. See DOI: 10.1039/c5tc01509j

\# Present address: Laboratoire CRISMAT UMR 6508 CNRS ENSICAEN, 6 Boulevard du Maréchal Juin, 14050 Caen, France.
}

efficiently scatter phonons, their impact on the charge carrier propagation is minimal when the domains are intergrown endotactically. This leads to the phonon glass electron crystal (PGEC) concept, which assumes a maximum $Z T$ in materials with glass-like thermal conductivity in combination with a high charge carrier mobility. The latter is mainly determined by the slope of conduction bands, with flat bands leading to a low charge carrier mobility but a high Seebeck coefficient. ${ }^{4}$ Much research has focused on skutterudite-type $\mathrm{CoSb}_{3}$ (space group $\operatorname{Im} \overline{3}$ ) as a PGEC material due to its favorable crystal structure. It can be considered a severely distorted $\mathrm{ReO}_{3}$ type with voids in the center of a $\left(\mathrm{CoSb}_{6 / 2}\right)_{8}$ unit large enough for the incorporation of loosely bound "rattling" atoms. Unfilled and undoped $\mathrm{CoSb}_{3}$ suffers from its rather high thermal conductivity, which is around $10 \mathrm{~W} \mathrm{~m}^{-1} \mathrm{~K}^{-1}$ at room temperature (RT). ${ }^{5}$ Whereas void filling with various heavy atoms can significantly reduce $\kappa_{\mathrm{ph}},{ }^{6-8}$ the electronic structure can be tuned by $\mathrm{Sb}$ substitution in the characteristic $\mathrm{Sb}_{4}$ units, for example by GeTe. ${ }^{9-11}$ Quenched germanium antimony tellurides (GST materials), on the other hand, exhibit a remarkably low thermal conductivity due to pronounced nanostructures. Upon slow cooling, their rocksalt-type high-temperature (HT) modification undergoes a phase transition to a trigonal layered one stable at RT. ${ }^{12,13}$ The layers are separated by van der Waals gaps, whereas vacancies 
in the cubic phase are randomly distributed on the cation position. When the HT phase is quenched, cation diffusion is limited, which leads to finite defect layers in a pseudocubic modification as found by high resolution transmission electron microscopy (HRTEM). ${ }^{14,15}$

The thermoelectric properties of skutterudites can be improved by nanoscale heterostructuring, for example with precipitates of $\mathrm{AgSbTe}_{2} \cdot{ }^{16}$ As this is unexpected in a classical mixture according to effective medium theory, ${ }^{17}$ this improvement is attributed to phonon scattering at nanoscale interfaces. In such heterogeneous systems, the transport properties need to be considered a combined result of the compounds present. Previous work aimed at decoupling the interdependent Seebeck coefficient and electrical conductivity. Thus a simultaneous increase of both properties may be feasible by a high charge carrier mobility in combination with interface energy filtering. ${ }^{16}$ To that effect, a combination of skutterudites with GST materials is intriguing as both exhibit promising thermoelectric properties; and similar atom environments in both compounds might lead to endotactic intergrowth.

\section{Experimental procedure}

\section{Synthesis}

All syntheses started from stoichiometric quaternary melts of the pure elements Co (99.995\%, Sigma Aldrich), Sb (99.9999\%, Smart Elements), Ge (99.999\%, Smart Elements) and Te (puriss., VEB Spurenmetalle Freiberg and 99.999\%, Alfa Aesar) in sealed silica glass ampoules under a dry Ar atmosphere. The samples were quenched after at least $2 \mathrm{~h}$ at $950{ }^{\circ} \mathrm{C}$ and subsequently annealed at $590{ }^{\circ} \mathrm{C}(0.5-4 \mathrm{~h})$ or at $530{ }^{\circ} \mathrm{C}(64.5 \mathrm{~h})$, followed again by quenching. Quenching rates were approximately $10 \mathrm{~K} \mathrm{~s}^{-1}$. Detailed information on the thermal treatment of the individual samples is given in the $\mathrm{ESI} \dagger$ (Table S1). Disc-shaped samples for thermoelectric characterization were obtained by using flatbottomed silica glass ampoules. The ingots were polished using SiC grinding powder and sawn by a diamond wire saw when cuboid samples were necessary.

\section{Analytical methods}

The morphology and composition of the samples were examined by means of scanning electron microscopy (SEM) and energydispersive X-ray spectroscopy (EDX) using a Zeiss LEO 1530 microscope (acceleration voltage $20 \mathrm{kV}$ ) equipped with an EDX detector (INCA system, Oxford Instruments). The phase composition was assessed by powder X-ray diffraction (PXRD) using a Huber G670 diffractometer (Guinier geometry with imagingplate detector, $\mathrm{Ge}(111)$ monochromator) with $\mathrm{Cu}-\mathrm{K}_{\alpha 1}$ radiation $(\lambda=1.54051 \AA)$ for RT measurements and a similar instrument with $\mathrm{Mo}-\mathrm{K}_{\alpha 1}$ radiation $(\lambda=0.71073 \AA$ ) for temperature-dependent measurements. RT diffraction patterns were obtained from crushed samples on flat specimen holders; for temperaturedependent measurements, samples in glass capillaries under dry $\mathrm{Ar}$ were rotated in a ceramic heating cradle (direct heat transfer).
Finely ground samples on copper grids coated with a holey carbon film as well as polished slabs of samples dimpled and thinned using Ar-ions (Duo-Mill, GATAN) were used for transmission electron microscopy (TEM). Selected area electron diffraction (SAED), high-resolution transmission electron microscopy (HRTEM) and scanning transmission electron microscopy (STEM) were done on a Titan 80-300 (FEI, field emission gun, voltage $300 \mathrm{kV}$ ) with a TEMTOPS 30 EDX spectrometer (EDAX) and on a Philips CM-200 ( $\mathrm{LaB}_{6}$ cathode, $200 \mathrm{kV}$, super-twin lens) with an R-TEM 136-5 EDX detector (EDAX).

Single-crystal structure elucidation was done by microfocus synchrotron diffraction (beamline ID11, ESRF, Grenoble) ${ }^{18}$ on a Huber diffractometer with a FReLoN2K CCD detector (dynamical range $2^{16}$ ) as detailed below. ${ }^{19}$ The approximate beam size was $700 \mathrm{~nm} \times 1.5 \mu \mathrm{m}(\lambda=0.2946 \AA \hat{=} 42.1 \mathrm{keV})$. Further details of the crystal structure investigations may be obtained from the Fachinformationszentrum Karlsruhe, 76344 EggensteinLeopoldshafen, Germany (fax: (+49) 7247-808-666; e-mail: crysdata@fiz-karlsruhe.de) on quoting the deposition numbers CSD 429336 and 429337.

Thermal diffusivities were measured in Ar flow using an LFA457 MicroFlash (Netzsch) with an InSb detector. A Cowan heat loss correction was applied. ${ }^{20}$ Additional diffusivity measurements were performed under He atmosphere with a Linseis LFA1000 equipped with an InSb detector using the Dusza model for simultaneous heat loss and finite pulse corrections. ${ }^{21}$ The thermal conductivity $\kappa$ was calculated as the product of the thermal diffusivity, the density (derived by the weight and the volume determined by Archimedes' principle or measuring the dimensions of the sample) and the Dulong-Petit heat capacity. All values were averaged from 5 measurement points at each temperature and linearly interpolated. The electrical conductivity and the Seebeck coefficient $S$ were simultaneously measured under a He atmosphere with a Linseis LSR-3 instrument equipped with $\mathrm{NiCr} / \mathrm{Ni}$ and $\mathrm{Ni}$ contacts. For measurements of $S$, the polarity of the thermocouples was continuously reversed (bipolar setup). Further measurements of the electrical conductivity and Hall coefficient $\left(R_{\mathrm{H}}\right)$ measurements were done on an in-house built facility using the van der Pauw method in a reversible magnetic field up to $2 \mathrm{~T}^{22}$ The Hall carrier density $n_{\mathrm{H}}$ was obtained according to $n_{\mathrm{H}}=1 / e R_{\mathrm{H}}$, where e is the electron charge. Additional $S$ measurements were obtained using W-Nb thermocouples. Calculated $Z T$ values are assumed to exhibit an absolute uncertainty of $20 \%{ }^{23}$ For detailed information on the devices used for each sample $c f$. Table $\mathrm{S} 1$ in the ESI. $\dagger$

All computer programs and databases used are given in Section I of the ESI, $\dagger$ including the respective references.

\section{Results}

\section{Chemical and phase analysis}

All investigated samples quenched from quaternary $\mathrm{Co} / \mathrm{Ge} / \mathrm{Sb} / \mathrm{Te}$ melts do not contain skutterudite-type phases, but different cobalt germanides and elemental cobalt as side-phases ( $c f$. Fig. S3 in $\mathrm{ESI} \dagger$ ). Thus, they were annealed at $530-590{ }^{\circ} \mathrm{C}$, i.e. in the existence 
range of the rocksalt-type HT modification of GST. Subsequent quenching yields skutterudite-type precipitates, whose size strongly depends on the annealing time. Average sizes of $1 \mu \mathrm{m}$ or less were found when the samples were annealed for $30 \mathrm{~min}$, whereas sizes of 2-5 $\mu \mathrm{m}$ were predominant for longer annealing times. However, the dependence of the precipitate size on the annealing temperature is not particularly pronounced. In order to avoid nanoscopic precipitates for the reliable determination of the phase composition by Rietveld refinement, the samples discussed in this section were annealed for $4 \mathrm{~h}$. In all samples, $\mathrm{Sb}$ in the skutterudite types turned out to be partially substituted by GeTe, with the substitution degree depending on the GeTe content of the starting material. Especially for relatively large phase fractions of skutterudite-type compounds, this substitution needs to be taken into account in the starting composition in order to avoid the precipitation of elemental Ge. Although the degree of substitution is slightly varying, a substitution of $1 / 3$ of $\mathrm{Sb}$ in $\mathrm{CoSb}_{3}$ by GeTe proved to be a good starting point for most sample compositions. Thus, $\left[\mathrm{CoSb}_{2}(\mathrm{GeTe})_{0.5}\right]_{x}(\mathrm{GeTe})_{n} \mathrm{Sb}_{2} \mathrm{Te}_{3}$ is a reasonable nominal formula as in an alloy-like formula $\left[\mathrm{CoSb}_{2}(\mathrm{GeTe})_{0.5}\right]_{x}\left[(\mathrm{GeTe})_{n} \mathrm{Sb}_{2} \mathrm{Te}_{3}\right]_{1-x}$, $x$ would still not be a measure of the skutterudite-type fraction as the amount of GST phase is also determined by $n$. The skutterudite-type fraction is best quantified via the overall Co content. The variation of $n$ yields matrix phases with the $9 P-(\mathrm{GeTe})_{2} \mathrm{Sb}_{2} \mathrm{Te}_{3}$ type for $n=2$, whereas the (pseudo-)cubic modification is present in quenched samples with higher $n$. Values of $n \geq 15$ yield trigonal matrix phases whose average structures correspond to the GeTe type. ${ }^{13,24}$ Mixtures of different matrix phases such as $9 P-(\mathrm{GeTe})_{2} \mathrm{Sb}_{2} \mathrm{Te}_{3}$ and the rocksalttype modification occur for intermediate compositions (e.g. with $n=5$ ). Quantitative phase analyses for a series of these samples with an approximately constant fraction of the skutterudite-type phase are shown in Fig. 1. The GeTe content of the precipitates was determined from the lattice parameters of the skutterudite-type phase, based on the fact that the solid solution series $\mathrm{CoSb}_{3}-\mathrm{CoGe}_{1.5} \mathrm{Te}_{1.5}$ obeys Vegard's law. ${ }^{9}$ Reference samples of $\mathrm{CoSb}_{3}$ and $\mathrm{CoSb}_{2.5}(\mathrm{GeTe})_{0.25}$ were synthesized and yielded lattice parameters that are in good agreement with the published ones. Thus, site occupancies (i.e. the degree of substitution) from ref. 9 were used and not refined. The refined phase fractions are slightly lower than the expected $12.9 \mathrm{wt} \%$. These deviations can be attributed to the imprecision of the method. By means of EDX, no incorporation of Co into the matrix could be detected and its composition did not deviate from the nominal composition. As no shift of the matrix' lattice parameters was observed, the composition of the matrix was not refined but constrained to the one assumed in the nominal composition of the starting material. Obviously, the bias with respect to the slightly varying GeTe content of the precipitates is negligible. Further refinements including samples with a GeTe-type GST main phase as well as with a homogeneous $9 P-\mathrm{Pb}_{2} \mathrm{Sb}_{2} \mathrm{Se}_{5}$-type ${ }^{25}$ matrix are given in the ESI $\dagger$ (Fig. S1). An increase of the nominal skutterudite-type fraction beyond $\approx 15 \%$ yields side-phases such as cobalt germanides or elemental Co.
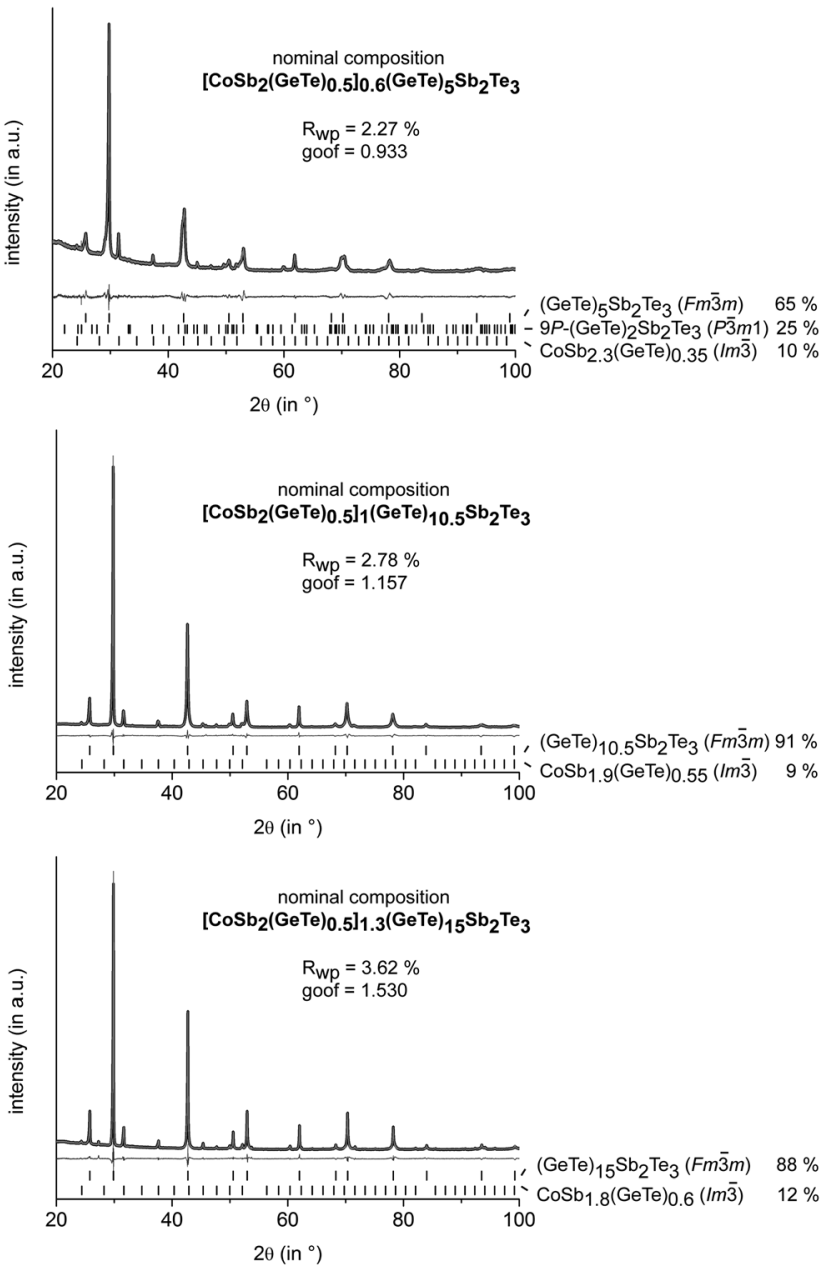

Fig. 1 PXRD patterns of different GST materials with skutterudite-type precipitates: experimental (black), calculated (light gray) and difference (dark gray) profiles; reflection positions are indicated as vertical lines; residuals and calculated phase fractions (weight\%) are given; structure models taken from ref. $26(P \overline{3} \mathrm{~m} 1), 27(\mathrm{Fm} \overline{3} \mathrm{~m})$ and $28(/ \mathrm{m} \overline{3})$; anisotropic peak broadening fitted using Stephens' model. ${ }^{29}$

\section{Temperature-dependent behavior}

Samples of $(\mathrm{GeTe})_{10.5} \mathrm{Sb}_{2} \mathrm{Te}_{3}$ quenched after $0.5 \mathrm{~h}$ at $590{ }^{\circ} \mathrm{C}$ exhibit cubic metrics. Temperature-dependent powder X-ray diffraction patterns show that upon heating a slight structure distortion towards a modification with a trigonal average structure sets in at $\sim 220{ }^{\circ} \mathrm{C}$ (T1 in Fig. 2), which explains the reflection broadening observed. At $\sim 425{ }^{\circ} \mathrm{C}(\mathrm{T} 2)$, the reflections become sharper again due to the phase transition to a rocksalt-type HT phase. Upon slow cooling, the reflection broadening sets in at $\sim 400{ }^{\circ} \mathrm{C}$ (T3). The phase transition at $\mathrm{T} 1$ is irreversible, thus no cubic phase is present at lower temperatures. In $\left[\mathrm{CoSb}_{2}(\mathrm{GeTe})_{0.5}\right]_{1}(\mathrm{GeTe})_{10.5} \mathrm{Sb}_{2} \mathrm{Te}_{3}$, the same phase transitions were found, but $\mathrm{T} 1$ is shifted towards higher temperatures $\left(\sim 275{ }^{\circ} \mathrm{C}\right)$ which leads to a narrower existence range of the trigonal modification. This impact of the precipitates on the phase transition temperatures of the matrix is possibly due to the additional domain boundaries which hinder cation diffusion. 

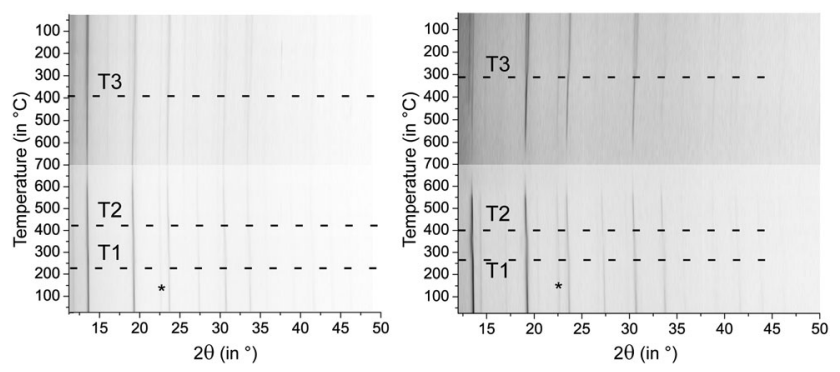

Fig. 2 Temperature-dependent PXRD patterns of samples with the nominal composition (GeTe) ${ }_{10.5} \mathrm{Sb}_{2} \mathrm{Te}_{3}$ (left) and $\left[\mathrm{CoSb}_{2}(\mathrm{GeTe})_{0.5}\right]_{1}(\mathrm{GeTe})_{10.5} \mathrm{Sb}_{2} \mathrm{Te}_{3}$ (right); phase transition temperatures are given as dashed lines (cf. discussion in the text); T1: pseudocubic - trigonal average structure; T2: trigonal rocksalt-type structure; T3: rocksalt-type - trigonal average structure; the reflection marked with an asterisk is caused by the furnace.

\section{Analysis of the micro-/nanostructure}

The particle size and distribution within the matrix were determined using SEM and STEM. A representative secondary electron image of a quenched and annealed (590 ${ }^{\circ} \mathrm{C}, 4 \mathrm{~h}$ ) sample with the nominal composition $\left[\mathrm{CoSb}_{2}(\mathrm{GeTe})_{0.5}\right]_{1}(\mathrm{GeTe})_{10.5} \mathrm{Sb}_{2} \mathrm{Te}_{3}$ as well as the STEM-EDX results are depicted in Fig. 3. The homogeneously distributed precipitates are 1-2 $\mu \mathrm{m}$ in size. Ge and Te are found in all regions of the sample, which confirms the $\mathrm{Sb}$ substitution in the skutterudite type as observed by the shift of the reflections in the PXRD patterns. Point EDX measurements agree well with the nominal composition (the complete EDX mapping as well point measurement results are given in Fig. S4 and Table S2 in the ESI $\dagger$ ). The coincidence of the $\langle 110\rangle^{*}$ direction of $\operatorname{CoSb}_{1.6}(\mathrm{GeTe})_{0.7}$ and the $\langle 100\rangle^{*}$ direction (indexing according to cubic metrics) of the matrix as observed for some crystallites hints at endotactic intergrowth (see Fig. S5 and S6 in the ESI $\dagger$ ). However, as the lattice parameters and thus the common atomic distances vary with the

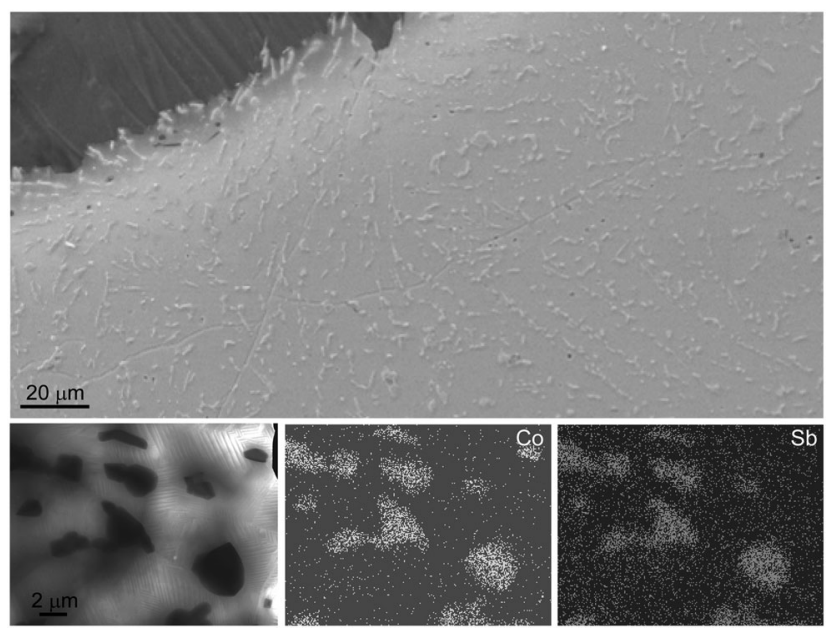

Fig. 3 SEM image of an Ar-ion-thinned sample with the nominal composition $\left[\mathrm{CoSb}_{2}(\mathrm{GeTe})_{0.5}\right]_{1}(\mathrm{GeTe})_{10.5} \mathrm{Sb}_{2} \mathrm{Te}_{3}$ (secondary electrons, top) and STEM-EDX element mapping of a sample region (bottom); dark-field image (left) and spatially resolved element distribution for $\mathrm{Co}$ and $\mathrm{Sb}$ (middle and right, respectively).
GeTe content $n$ of the GST phase as well as with the $\mathrm{Sb}$ substitution degree in the skutterudite-type phase, no oriented intergrowth was found in general.

SEM imaging revealed no significant porosity, neither of quenched samples (see the image of polished ingot in Fig. 3), nor after several heating cycles. There are no pronounced changes in the microstructure upon such a thermal treatment. Although the materials are heterogeneous, the high density of the compact samples indicates that the transport properties discussed below are not considerably affected by extrinsic effects such as pores. The theoretical densities (weighted average of the components) vary from $6.252 \mathrm{~g} \mathrm{~cm}^{-3}$ for pure $(\mathrm{GeTe})_{10.5} \mathrm{Sb}_{2} \mathrm{Te}_{3}$ to $6.477 \mathrm{~g} \mathrm{~cm}^{-3}$ for $\left[\mathrm{CoSb}_{2}(\mathrm{GeTe})_{0.5}\right]_{2}(\mathrm{GeTe})_{10.5} \mathrm{Sb}_{2} \mathrm{Te}_{3}$, and typical measured values are $>95 \%$ of the latter.

\section{Structure elucidation}

Due to the heterogeneity of the material as well as the microscale size of the precipitates, conventional X-ray methods are not suitable for structure elucidation. Thus, a discrete skutteruditetype crystallite in a polished and thinned slab cut from an ingot was selected by means of TEM. The type and composition of the precipitate and the surrounding matrix were confirmed by SAED and EDX. This crystallite was optically re-located at the European Synchrotron Radiation Facility (ESRF) and centered by means of fluorescence scans for $\mathrm{Co}, \mathrm{Sb}$ and $\mathrm{Te}$ (for a detailed description of this procedure, see ref. 30). Single-crystal datasets of the precipitates as well as the surrounding matrix were collected using a $700 \mathrm{~nm} \times 1.5 \mu \mathrm{m}$ beam. The results of the structure refinements are given in Table 1 and Tables S4-S7 (ESI $\dagger$ ). In the skutterudite-type precipitates, the characteristic $\mathrm{Sb}_{4}$ units (highlighted in Fig. 4) are more distorted than in $\mathrm{CoSb}_{3}$. Two types of bonds can be distinguished, which both are shorter than in $\mathrm{CoSb}_{3}$ (cf. Fig. 4: 2.7911(7) vs. $2.8947 \AA$ and 2.9352(7) $\AA$ vs. $2.9796 \AA$, ratio $0.95: 1$ vs. $0.97: 1)$. This distortion corresponds to the Sb substitution by GeTe (as also indicated by shifted reflection positions in powder diffraction patterns, see above) due to the smaller atomic radii of Ge and Te $(1.25 \text { and } 1.40 \AA \text { vs. } 1.45 \AA \text { for } \mathrm{Sb})^{31}$ and has a significant influence on the band structure. ${ }^{32,33}$ No residual electron density was found in the voids, thus no significant void filling by Te or Ge is present.

While the PXRD pattern shows pronounced rhombohedral reflection splitting of the matrix ( $c f$. Fig. S2 in the ESI $\dagger$ ), the region analyzed with microfocused synchrotron radiation seemed to be very close to cubic metrics. This is due to twinning of rhombohedral crystals whose reflection positions correspond to averaged superimposed groups of reflections according to a symmetry reduction from $F m \overline{3} m$ to $R \overline{3} m$ (a translationengleiche subgroup of index 4). A further translationengleiche reduction (index 2) leads to the GeTe-type $(R 3 m)$ as confirmed by refinements that showed a pronounced distortion of the GeTe-type layers along the cubic $\langle 111\rangle$ direction. The deviation of the cations' $z$ parameter from zero on the Wyckoff position $3 a$ : 0,0 , $0.0203(12)$ is significant by more than $10 \sigma$. Thus, pseudomerohedral fourfold twinning was taken into account while a possible additional inversion twinning in the polar space group 
Table 1 Crystallographic data and structure refinements of skutterudite-type $\mathrm{CoSb}_{1.53}(\mathrm{GeTe})_{0.74}$ and $\mathrm{Ge}_{0.77} \mathrm{Sb}_{0.15} \mathrm{Te}_{1}=\left(\mathrm{GeTe}_{10.3} \mathrm{Sb}_{2} \mathrm{Te}_{3}\right.$ at RT

\begin{tabular}{|c|c|c|}
\hline Formula & $\mathrm{CoSb}_{1.53(9)}(\mathrm{GeTe})_{0.74(5)}$ & $\mathrm{Ge}_{0.77} \mathrm{Sb}_{0.15} \mathrm{Te}_{1}=(\mathrm{GeTe})_{10.3} \mathrm{Sb}_{2} \mathrm{Te}_{3}^{a}$ \\
\hline Formula mass $\left(\mathrm{g} \mathrm{mol}^{-1}\right)$ & 393.6 & 202.2 \\
\hline Crystal system/space group (no.) & Cubic/Im̄ $\overline{3}(204)$ & Trigonal/R3m (160) \\
\hline Cell parameters (in $\AA$ ) & $a=8.9166(3)$ & $\begin{array}{l}a=4.2348(11) \\
c=10.373(3)\end{array}$ \\
\hline Cell volume (in $\AA^{3}$ ) & $708.93(6)$ & $161.10(11)$ \\
\hline $\mathrm{X}$-ray density (in $\mathrm{g} \mathrm{cm}^{-3}$ ) & 7.375 & 6.252 \\
\hline Formula units (per unit cell) & 8 & 3 \\
\hline$d_{\min }($ in $\AA)$ & 0.50 & 0.55 \\
\hline Absorption coefficient (in $\mathrm{mm}^{-1}$ ) & 10.27 & 9.05 \\
\hline Absorption correction & \multicolumn{2}{|c|}{ Semiempirical (SADABS) ${ }^{36}$} \\
\hline Reflections measured/independent & $4064 / 562$ & $871 / 138$ \\
\hline$R_{\mathrm{int}} / R_{\sigma}$ & $0.0521 / 0.0334$ & 0.0797 \\
\hline Parameters/restraints & $10 / 0$ & $9 / 0$ \\
\hline Twin fractions & - & $0.213(18), 0.252(13), 0.254(13), 0.281^{b}$ \\
\hline
\end{tabular}

${ }^{a}$ Fixed composition from SEM point measurements ( $c f$. Table S2 in the ESI). ${ }^{b}$ Transformation matrix from the rocksalt-type setting to one

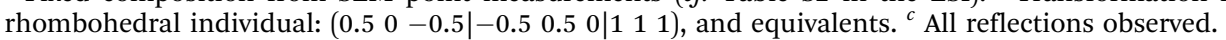

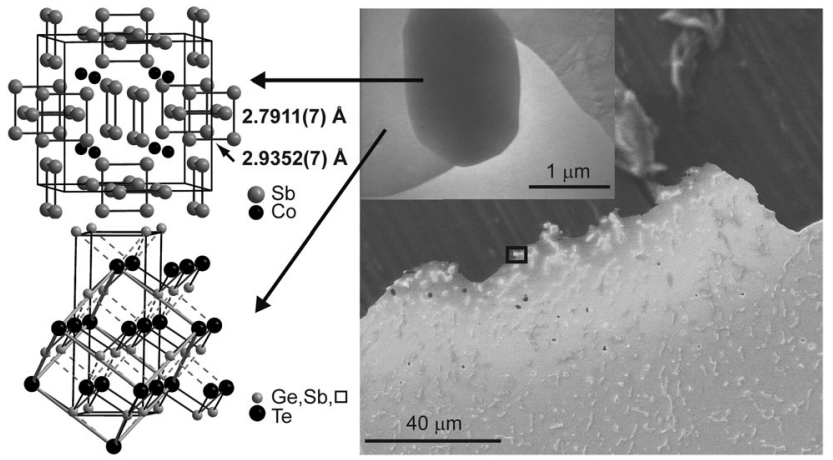

Fig. 4 Structure elucidation of $\left[\mathrm{CoSb}_{2}(\mathrm{GeTe})_{0.5}\right]_{1}(\mathrm{GeTe})_{10.5} \mathrm{Sb}_{2} \mathrm{Te}_{3}$ by microfocus X-ray diffraction; SEM image of polished and Ar-ion thinned sample (right) and examined crystallite (STEM image, inset, the corresponding area is marked by the small black box in the SEM image); structures of the skutterudite-type crystallite (top left) and the distorted rocksalt-type GST matrix (GeTe type, bottom left); characteristic anion-anion distances in distorted squares of skutterudite-type are given.

$R 3 m$ was neglected as it did not change the structure model but rendered the refinement unstable. This twinning is typical for germanium antimony tellurides and was found to play an important role in their phase transition behavior by inducing stress and strain, especially in small crystallites. ${ }^{34,35}$

\section{Thermoelectric properties}

The characteristic phase transitions of GST materials lead to a pronounced discrepancy of the thermoelectric properties between the first and the following heating curves. Fig. 5 shows the results of the first measurement cycles of the Seebeck coefficient and the electrical conductivity for $(\mathrm{GeTe})_{10.5} \mathrm{Sb}_{2} \mathrm{Te}_{3}$ in comparison to $\left[\mathrm{CoSb}_{2}(\mathrm{GeTe})_{0.5}\right]_{0.5}(\mathrm{GeTe})_{10.5} \mathrm{Sb}_{2} \mathrm{Te}_{3}$. Upon heating the quenched material, extrema around $250{ }^{\circ} \mathrm{C}$ can be correlated with T1 in Fig. 2 and are attributed to the transition of the
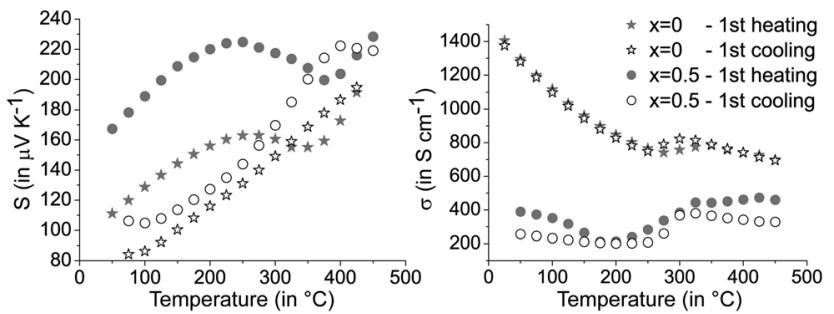

Fig. 5 Seebeck coefficients $S$ (left) and electrical conductivities $\sigma$ (right) of $\left[\mathrm{CoSb}_{2}(\mathrm{GeTe})_{0.5}\right]_{x}(\mathrm{GeTe})_{10.5} \mathrm{Sb}_{2} \mathrm{Te}_{3}$ with $x=0$ and 0.5 ; first heating and subsequent slow cooling of quenched samples.

metastable quenched GST phase to the stable trigonal layered one. The higher phase transition temperature of the heterostructured sample is in good agreement with temperature-dependent PXRD measurements (see above). Above $400{ }^{\circ} \mathrm{C}$, the transition to the rocksalt-type HT modification (T2 in Fig. 2) also influences the charge transport as indicated by the changing slope of the measurement curves (Fig. 5). Upon cooling, the layered phase is re-formed but no phase transition back to a (pseudo-) cubic modification is observed. ${ }^{37,38}$ Thus, the cooling curve as well as the following measurement cycles show only the transition trigonal-cubic (heating) and cubic-trigonal (cooling). This reversible structural process results in a reproducible hysteresis of each heating and cooling curve, except the first heating curve of quenched samples. The following discussion thus focusses on cooling curves, which do not significantly change during consecutive cycles. The corresponding heating curves (except the very first one) show the same trends. Selected plots for consecutive measurement cycles are given in Fig. S7 in the ESI. $\dagger$

The precipitation of $\mathrm{CoSb}_{2}(\mathrm{GeTe})_{0.5}$ in $(\mathrm{GeTe})_{10.5} \mathrm{Sb}_{2} \mathrm{Te}_{3}$ leads to significantly higher Seebeck coefficients and lower electrical conductivities (cf. Fig. 6) than those of pure $(\mathrm{GeTe})_{10.5} \mathrm{Sb}_{2} \mathrm{Te}_{3}$. This effect is most pronounced for rather low fractions of $\mathrm{CoSb}_{2}(\mathrm{GeTe})_{0.5}$. 

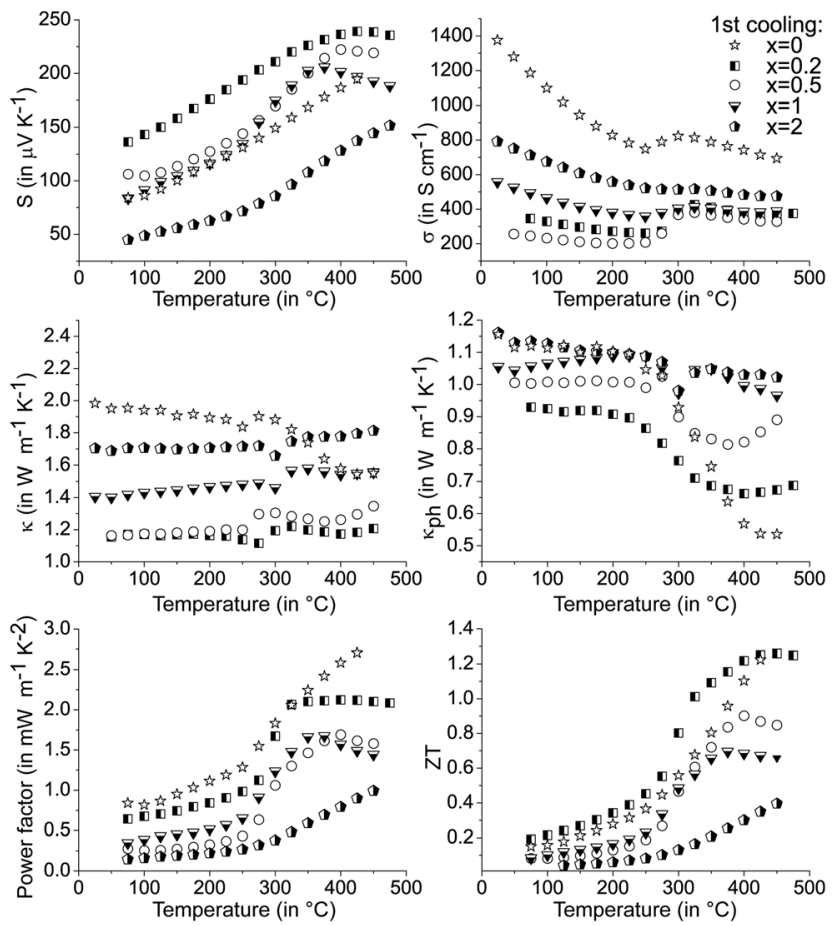

Fig. 6 Thermoelectric properties of $\left[\mathrm{CoSb}_{2}(\mathrm{GeTe})_{0.5}\right]_{x}(\mathrm{GeTe})_{10.5} \mathrm{Sb}_{2} \mathrm{Te}_{3}$ (first cooling curves measured after heating quenched samples to $500{ }^{\circ} \mathrm{C}$ ): Seebeck coefficients (S, top left), electrical conductivities ( $\sigma$, top right); total ( $\kappa$, middle left) and phononic ( $\kappa_{\mathrm{ph}}$, middle right) thermal conductivities calculated with the following Lorenz numbers (cf. text, in $10^{-8} \mathrm{~W} \Omega \mathrm{K}^{-2}$ ): $2.02(x=0), 2.00(x=0.2), 1.92(x=0.5), 2.10(x=1)$ and $2.30(x=2)$; power factors $S^{2} \sigma$ (bottom left) and $Z T$ values (bottom right).

As a result, the power factor $S^{2} \sigma$ of $\left[\mathrm{CoSb}_{2}(\mathrm{GeTe})_{0.5}\right]_{0.2}(\mathrm{GeTe})_{10.5} \mathrm{Sb}_{2} \mathrm{Te}_{3}$ is comparable to the one found for $(\mathrm{GeTe})_{10.5} \mathrm{Sb}_{2} \mathrm{Te}_{3}$. An increase of the precipitate content leads to electrical conductivities approaching the values of pure (GeTe) ${ }_{10.5} \mathrm{Sb}_{2} \mathrm{Te}_{3}$, but this effect is over-compensated by a simultaneous decrease of the Seebeck coefficient, which leads to power factors lower than those of $(\mathrm{GeTe})_{10.5} \mathrm{Sb}_{2} \mathrm{Te}_{3}$.

In several (but not all) Seebeck coefficient curves, broad maxima could indicate the activation of minority carriers. Nevertheless, changes in the defect content as a consequence of phase transitions are also likely to influence the Seebeck coefficient via changes in the charge carrier concentration (as evident from Hall measurements, see below). As a tendency, these maxima are found at lower temperatures for higher precipitate contents, e.g., they shift from $\sim 425{ }^{\circ} \mathrm{C}$ for $\left[\mathrm{CoSb}_{2}(\mathrm{GeTe})_{0.5}\right]_{0.2}(\mathrm{GeTe})_{10.5} \mathrm{Sb}_{2} \mathrm{Te}_{3}$ to $\sim 375{ }^{\circ} \mathrm{C}$ for $\left[\mathrm{CoSb}_{2}(\mathrm{GeTe})_{0.5}\right]_{1}(\mathrm{GeTe})_{10.5} \mathrm{Sb}_{2} \mathrm{Te}_{3}$. The thermal band gap $\left(E_{\mathrm{g}}\right)$ as very roughly determined from the maximum Seebeck coefficients using the Goldsmid-Sharp relation $^{39} S_{\max } \approx E_{\mathrm{g}} / 2 e T_{\max }$ (with the elementary charge $e$ ) amounts to $\sim 0.3 \mathrm{eV}$. There is no such Seebeck maximum for $\left[\mathrm{CoSb}_{2}(\mathrm{GeTe})_{0.5}\right]_{2}(\mathrm{GeTe})_{10.5} \mathrm{Sb}_{2} \mathrm{Te}_{3}$ and $(\mathrm{GeTe})_{10.5} \mathrm{Sb}_{2} \mathrm{Te}_{3}$ itself. Differences between pure (GeTe) ${ }_{10.5} \mathrm{Sb}_{2} \mathrm{Te}_{3}$ and the heterostructured samples might be due to slight Co doping in the matrix of the heterostructured materials below the detection limit of EDX analysis.

These findings motivate a closer look on the electrical transport properties of heterostructured $\left[\mathrm{CoSb}_{2}(\mathrm{GeTe})_{0.5}\right]_{x}(\mathrm{GeTe})_{10.5} \mathrm{Sb}_{2} \mathrm{Te}_{3}$
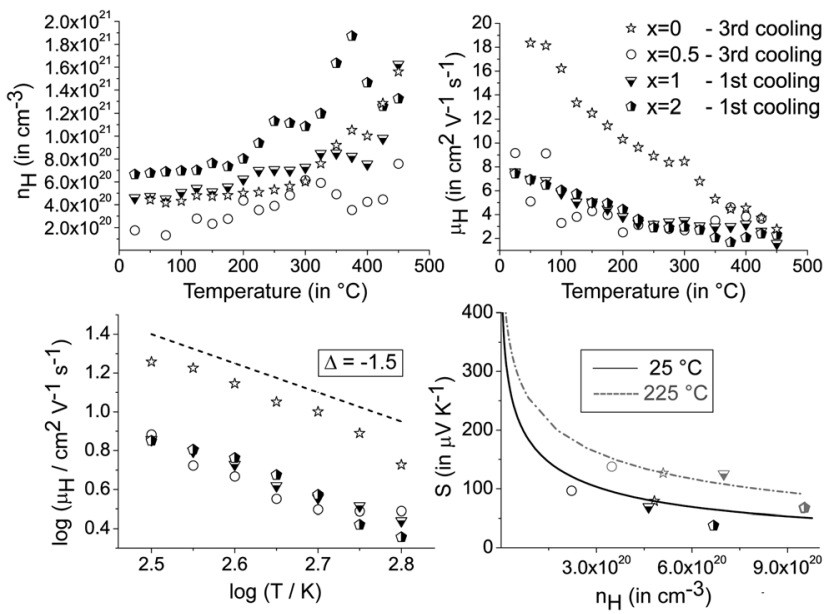

Fig. 7 Hall carrier concentrations ( $n_{\mathrm{H}}$, top left) and mobilities ( $\mu_{\mathrm{H}}$, top right) of $\left[\mathrm{CoSb}_{2}(\mathrm{GeTe})_{0.5}\right]_{x}(\mathrm{GeTe})_{10.5} \mathrm{Sb}_{2} \mathrm{Te}_{3}$ measured after heating to $500{ }^{\circ} \mathrm{C}$ (third cooling curve for $x=0$ and $x=0.5$ and first one for $x=1$ and $x=2$, respectively) as well as decrease of the Hall mobility as a function of temperature (bottom left, data smoothed using a Savitzky-Golay filter; a line with a slope of $\Delta=-1.5$ is indicated); Seebeck coefficient $S$ as a function of the Hall carrier concentration (Pisarenko plot) as predicted by the SPB model (bottom right) at $25{ }^{\circ} \mathrm{C}$ (solid line) and $225{ }^{\circ} \mathrm{C}$ (dashed line) with $m^{*}=2.5 m_{e}\left(25^{\circ} \mathrm{C}\right)$ and $2.75 m_{e}\left(225{ }^{\circ} \mathrm{C}\right)$ as calculated using $S$ and $n_{\mathrm{H}}$ measured for the sample with $x=0$ (black symbols are measurement points at $25^{\circ} \mathrm{C}$, gray ones at $225^{\circ} \mathrm{C}$.

materials. All samples exhibit p-type Hall carrier concentrations of $10^{20}-10^{21} \mathrm{~h}^{+} \mathrm{cm}^{-3}$, which are in the range of typical heavily doped semiconductors. In Fig. 7, the data of the Hall mobility are smoothed using a Savitzky-Golay filter, while the original data show some scattering due to contact resistances in the van der Pauw setup (a plot of the raw data is given in the $\mathrm{ESI} \dagger$ ). In all samples, the Hall mobility decrease with temperature approximately follows a $T^{3 / 2}$ relationship. This behavior implies that acoustic phonons are the primary scattering source that limits the charge carrier mobility. As a consequence, the single parabolic band (SPB) model with the acoustic phonon scattering approximation is applicable in the temperature range with an approximately linear increase of the Seebeck coefficient and electrical resistivity, i.e. up to $250{ }^{\circ} \mathrm{C}$. The effective charge carrier mass $m^{*}$ is determined by the measured Hall carrier density $n_{\mathrm{H}}=n_{\mathrm{c}} / r_{\mathrm{H}}$ (assuming a spherical Fermi surface, $n_{\mathrm{c}}=$ chemical carrier concentration, $r_{\mathrm{H}}=$ calculated Hall factor) using

$$
n_{\mathrm{c}}=4 \pi\left(2 m^{*} k_{\mathrm{B}} T / h^{2}\right)^{3 / 2} F_{1 / 2}(\eta)
$$

and

$$
r_{\mathrm{H}}=3 F_{1 / 2}(\eta) F_{-1 / 2}(\eta) / 4 F_{0}(\eta)^{2}
$$

with the reduced chemical potential $\eta$ calculated from the measured Seebeck coefficient

$$
S=k_{\mathrm{B}} / e\left(2 F_{1}(\eta) / F_{0}(\eta)-\eta\right)
$$

The Fermi integrals $F_{x}(\eta)$ are

$$
F_{x}(\eta)=\int f \varepsilon^{x} \mathrm{~d} \varepsilon=\int \varepsilon^{x} \mathrm{~d} \varepsilon /(1+\exp [\varepsilon-\eta]) .
$$


Fig. 7 also shows the Seebeck coefficient as a function of the Hall carrier concentration (Pisarenko plot) as calculated from the measured Hall mobility and Seebeck values of $(\mathrm{GeTe})_{10.5} \mathrm{Sb}_{2} \mathrm{Te}_{3}$ using the SPB model. The effective masses used are $2.5 m_{\mathrm{e}}$ for the $25{ }^{\circ} \mathrm{C}$ plot and $2.75 m_{\mathrm{e}}$ for the $225{ }^{\circ} \mathrm{C}$ plot, respectively, as calculated using $S$ and $n_{\mathrm{H}}$ measured for the sample with $x=0$. As is typical for single parabolic band behavior, the measured Seebeck coefficients for heterostructured $\left[\mathrm{CoSb}_{2}(\mathrm{GeTe})_{0.5}\right]_{x}(\mathrm{GeTe})_{10.5} \mathrm{Sb}_{2} \mathrm{Te}_{3}$ samples are slightly lower than the calculated ones. ${ }^{40}$ The lower charge carrier mobilities for heterostructured materials up to $375{ }^{\circ} \mathrm{C}$ are in accordance with the higher Seebeck coefficients and the lower electrical conductivities in comparison to pure (GeTe) ${ }_{10.5} \mathrm{Sb}_{2} \mathrm{Te}_{3}$.

The mobility curves of samples with and without precipitates meet at $375{ }^{\circ} \mathrm{C}$; however, in most heterostructured materials, lower charge carrier concentrations yield lower electrical conductivities than in $(\mathrm{GeTe})_{10.5} \mathrm{Sb}_{2} \mathrm{Te}_{3}$. For example, the charge carrier concentration of $\left[\mathrm{CoSb}_{2}(\mathrm{GeTe})_{0.5}\right]_{0.5}(\mathrm{GeTe})_{10.5} \mathrm{Sb}_{2} \mathrm{Te}_{3}$ is roughly half that of $(\mathrm{GeTe})_{10.5} \mathrm{Sb}_{2} \mathrm{Te}_{3}$, but more skutterudite-type precipitates lead to an increase of the charge carrier concentration. Note that minority carrier transport sets in above $\sim 250{ }^{\circ} \mathrm{C}$. The Hall factor thus consists of contributions of both carrier types at elevated temperatures, which were not investigated in detail. In order to determine the ideal charge carrier concentration, the maximum $Z T$ with respect to the Hall carrier concentration was modelled ( $c f$. Fig. 8) under the assumption of SPB behavior using

$$
Z T=S^{2} /\left(L+(\psi \beta)^{-1}\right)
$$

with the $\beta$ parameter

$$
\beta=\mu_{0}\left(m^{*} / m_{\mathrm{e}}\right)^{3 / 2} T^{5 / 2} / \kappa_{\mathrm{ph}}
$$

which includes the mobility parameter

$$
\mu_{0}=\mu_{\mathrm{H}} F_{-1 / 2}(\eta) / 2 F_{0}(\eta)
$$

and the $\psi$ function

$$
\psi=8 / 3 \pi e\left(2 m_{\mathrm{e}} k_{\mathrm{B}} / h^{2}\right)^{3 / 2} F_{0}(\eta) .
$$

$L$ is the Lorenz number and is given by

$$
L=k_{\mathrm{B}}{ }^{2} / e^{2}\left(3 F_{0}(\eta) F_{2}(\eta)-4 F_{1}(\eta)^{2}\right) / F_{0}(\eta)^{2} .
$$

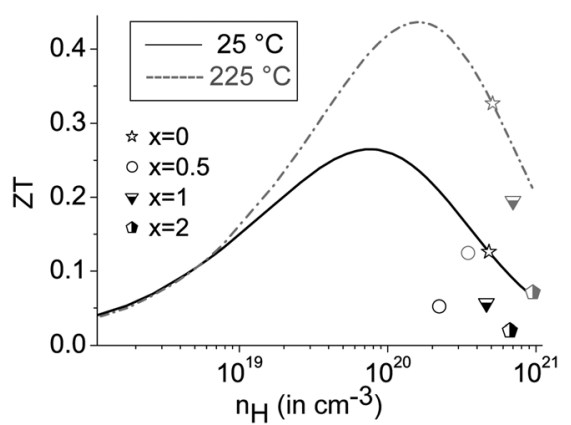

Fig. 8 Calculated $Z T$ as a function of Hall carrier concentration $n_{H}$ (lines) at $25{ }^{\circ} \mathrm{C}$ and $225^{\circ} \mathrm{C}$; measured values are given as symbols (see legend); the parameters used at $25^{\circ} \mathrm{C}$ (black data) and $225^{\circ} \mathrm{C}$ (gray data) were $m^{\star}=$ 2.5 and $2.75 \mathrm{~m}_{\mathrm{e}}, \kappa_{\mathrm{ph}}=1.21$ and $1.22 \mathrm{~W} \mathrm{~m}^{-1} \mathrm{~K}^{-1}$, and intrinsic mobilities of 18.0 and $9.6 \mathrm{~cm}^{2} \mathrm{~V}^{-1} \mathrm{~s}^{-1}$ as measured for $(\mathrm{GeTe})_{10.5} \mathrm{Sb}_{2} \mathrm{Te}_{3}$, respectively.
The predicted $Z T_{\max }$ at $25{ }^{\circ} \mathrm{C}$ is 0.26 for a Hall carrier concentration $n_{\mathrm{H}}=8.0 \times 10^{19} \mathrm{~h}^{+} \mathrm{cm}^{-3}$ and reaches 0.44 at $225{ }^{\circ} \mathrm{C}$ for $n_{\mathrm{H}}=1.6 \times 10^{20} \mathrm{~h}^{+} \mathrm{cm}^{-3}$. The experimental results for the heterostructured materials are lower than the calculated $Z T$ values for their carrier concentrations, which is mostly due to lower charge carrier mobilities than those used for calculation (which are the measured values for $\left.(\mathrm{GeTe})_{10.5} \mathrm{Sb}_{2} \mathrm{Te}_{3}\right)$. All the samples are overdoped with respect to the Hall carrier concentrations, but all are in the same $n_{\mathrm{H}}$ range.

The thermal conductivitites were calculated using the DulongPetit heat capacity $C_{\mathrm{p}}$. Measured $C_{\mathrm{p}}$ values for the single components are close to these values. ${ }^{14,38,41}$ A study on GST materials (see Fig. S9 in the ESI $\dagger$ ) showed deviations from the ideal values, especially at elevated temperatures, which are within the range of the reliability interval of the measurements. The error in $Z T$ introduced by the Dulong-Petit approximation can therefore be considered only a few percent in the present cases. All thermal conductivity curves show pronounced minima above $250{ }^{\circ} \mathrm{C}$, which hints at the onset of diffusion phenomena ( $c f$. Fig. 6 middle). Small fractions of skutterudite-type precipitates yield a thermal conductivity which is much lower than that of $(\mathrm{GeTe})_{10.5} \mathrm{Sb}_{2} \mathrm{Te}_{3}$ without precipitates, but $\kappa$ increases upon higher skutterudite-type fractions which correlates with the increase of the electrical conductivity. For $\kappa_{\mathrm{ph}}$ calculations, individual Lorenz numbers for each composition were determined at RT assuming SPB behavior. Up to $275{ }^{\circ} \mathrm{C}$, the lattice thermal conductivity is significantly reduced for heterostructured material and almost temperature-independent, which might be due to a large residual optical phonon contribution. ${ }^{42}$

The curve discontinuities of the single parameters compensate each other, which leads to $Z T$ curves that appear with almost no discontinuities (Fig. 6 bottom). For small fractions of skutterudite-type precipitates and most pronounced at intermediate temperatures, there is an apparent increase of $Z T$ upon heterostructuring (30\% in certain temperature ranges) which due to the hysteresis and microstructure evolution has considerable uncertainty. The increase in $Z T$ is mainly due to a lower thermal conductivity in combination with only a slightly lower power factor.

\section{Conclusions}

This study shows that heterostructures with nanoscale skutteruditetype precipitates are an intriguing way of enhancing the thermoelectric properties of germanium antimony tellurides by means of decreasing their thermal conductivities. Further improvement of the $Z T$ values might be achieved by tuning the charge carrier concentration, which is rather high in the samples investigated. It could be reduced by doping the precipitates with n-type carriers. If these are induced by filling the cage-like voids of the skutterudite type, there may be additional influence on $\kappa$. Nevertheless, slightly lower maximum $Z T$ values upon precipitation of skutterudite-type crystallites might be acceptable for the sake of broader existence ranges of the single modifications of the matrix, which thus results in an improved cyclability 
of GST materials. Due to the complex phase transitions that occur upon heating, the phase composition needs to be carefully controlled, especially regarding the thermal treatment during sample preparation. For a deeper understanding of the phase transitions involved, a detailed analysis of the phase contents is crucial. For this, microfocus synchrotron diffraction is the method of choice, as small crystalline precipitates can be examined without the shortcomings of electron microscopy.

Further studies might include ball-milled heterogeneous material, where the size control of the precipitates is straightforward and an additional reduction of the thermal conductivity is expected.

\section{Acknowledgements}

Financial support from the European Union (European Social Fund, NFG "Effiziente Energienutzung: Neue Konzepte und Materialien") and the Deutsche Forschungsgemeinschaft (Grant OE530/1-2) is gratefully acknowledged. The authors thank Dr Gavin B. Vaughan, Dr Jonathan Wright and Dr Loredana Erra (ESRF, Grenoble) for their support during the synchrotron measurements (project HS-4625). We are indebted to Prof. Dr Wolfgang Schnick (LMU Munich) for his generous support of this work. Tom Faske, Christina Fraunhofer and Sabine Hübner are acknowledged for help with some preliminary syntheses. GJS acknowledges support of DoE EFRC-S3TEC Award DE-SC0001299.

\section{References}

1 E. Quarez, K.-F. Hsu, R. Pcionek, N. Frangis, E. K. Polychroniadis and M. G. Kanatzidis, J. Am. Chem. Soc., 2005, 127, 9177.

2 K. Hoang, K. Desai and S. D. Mahanti, Phys. Rev. B: Condens. Matter Mater. Phys., 2005, 72, 064102.

3 M. G. Kanatzidis, Chem. Mater., 2010, 22, 648.

4 Y. Pei, H. Wang and G. J. Snyder, Adv. Mater., 2012, 24, 6125.

5 J. R. Sootsman, D. Young Chung and M. G. Kanatzidis, Angew. Chem., Int. Ed., 2009, 48, 8616.

6 G. S. Nolas, D. T. Morelli and T. M. Tritt, Annu. Rev. Mater. Sci., 1999, 29, 89.

7 G. Tang, D. Zhang, G. Chen, F. Xu and Z. Wang, Solid State Commun., 2012, 152, 2193.

8 B. C. Sales, D. Mandrus, B. C. Chakoumakos, V. Keppens and J. R. Thompson, Phys. Rev. B: Condens. Matter Mater. Phys., 1997, 56, 15081.

9 B. Duan, P. Zhai, L. Liu and Q. Zhang, J. Electron. Mater., 2011, 40, 932.

10 X. Su, H. Li, Q. Guo, X. Tang, Q. Zhang and C. Uher, J. Electron. Mater., 2011, 40, 1286.

11 X. Su, H. Li, G. Wang, H. Chi, X. Zhou, X. Tang, Q. Zhang and C. Uher, Chem. Mater., 2011, 23, 2948.

12 M. N. Schneider, T. Rosenthal, C. Stiewe and O. Oeckler, Z. Kristallogr., 2010, 225, 463.
13 T. Matsunaga, H. Morita, R. Kojima, N. Yamada, K. Kifune, Y. Kubota, Y. Tabata, J.-J. Kim, M. Kobata, E. Ikenaga and K. Kobayashi, J. Appl. Phys., 2008, 103, 093511.

14 T. Rosenthal, M. N. Schneider, C. Stiewe, M. Döblinger and O. Oeckler, Chem. Mater., 2011, 23, 4349.

15 H. S. Lee, B.-S. Kim, C.-W. Cho, M.-W. Oh, B.-K. Min, S.-D. Park and H.-W. Lee, Acta Mater., 2015, 91, 83.

16 J. Peng, L. Fu, Q. Liu, M. Liu, J. Yang, D. Hitchcock, M. Zhou and J. He, J. Mater. Chem. A, 2014, 2, 73.

17 D. Stroud, Superlattices Microstruct., 1998, 23, 567.

18 G. B. M. Vaughan, J. P. Wright, A. Bytchkov, C. Curfs, C. Grundlach, M. Orlova, L. Erra, H. Gleyzolle, T. Buslaps, A. Götz, G. Suchet, S. Petitdemange, M. Rossat, L. Margulies, W. Ludwig, A. Snigirey, I. Snigireva, H. O. Sorensen, E. M. Lauridsen, U. L. Olsen, J. Oddershede and H. F. Poulsen, Challenges in Materials Science Possibilities in $3 D$ and $4 D$ Characterization Techniques: Proceedings of the 31st Risø International Symposium on Materials Science, 2010, 457.

19 J.-C. Labiche, O. Mathon, S. Pascarelli, M. A. Newton, G. G. Ferre, C. Curfs, G. Vaughan, A. Homs and D. F. Carreiras, Rev. Sci. Instrum., 2007, 78, 091301.

20 R. D. Cowan, J. Appl. Phys., 1963, 34, 926.

21 L. Dusza, High Temp. - High Pressures, 1995/1996, 27/28, 467. 22 K. A. Borup, E. S. Toberer, L. D. Zoltan, G. Nakatsukasa, M. Errico, J. P. Fleurial, B. B. Iversen and G. J. Snyder, Rev. Sci. Instrum., 2012, 83, 123902.

23 Y. Pei, X. Shi, A. LaLonde, H. Wang, L. Chen and G. J. Snyder, Nature, 2011, 473, 66.

24 T. K. Chattopadhyay, J. X. Boucherle and H. G. von Schnering, J. Phys. C: Solid State Phys., 1987, 20, 1431.

25 K. A. Agaev, A. G. Talybov and S. A. Semiletov, Sov. Phys. Crystallogr., 1967, 11, 630.

26 P. Urban, M. N. Schneider, L. Erra, S. Welzmiller, F. Fahrnbauer and O. Oeckler, CrystEngComm, 2013, 15, 4823.

27 T. Matsunaga, R. Kojima, N. Yamada, K. Kifune, Y. Kubota, Y. Tabata and M. Takata, Inorg. Chem., 2006, 45, 2235.

28 D. G. Mandrus, A. Migliori, T. W. Darling, M. F. Hundley, E. J. Peterson and J. D. Thompson, Phys. Rev. B: Condens. Matter Mater. Phys., 1995, 52, 4926.

29 P. W. Stephens, J. Appl. Crystallogr., 1999, 32, 281.

30 F. Fahrnbauer, T. Rosenthal, T. Schmutzler, G. Wagner, G. Vaughan, J. Wright and O. Oeckler, Angew. Chem., Int. Ed., 2015, 54, DOI: 10.1002/anie.201503657.

31 J. C. Slater, J. Chem. Phys., 1964, 41, 3199.

32 P. Vaqueiro, G. G. Sobany, A. V. Powell and K. S. Knight, J. Solid State Chem., 2006, 179, 2047.

33 D. Jung, M.-H. Whangbo and S. Alvarez, Inorg. Chem., 1990, 29, 2252.

34 P. Urban, M. N. Schneider, M. Seemann, J. P. Wright and O. Oeckler, Z. Kristallogr., 2015, 230, 369.

35 M. N. Schneider, P. Urban, A. Leineweber, M. Döblinger and O. Oeckler, Phys. Rev. B: Condens. Matter Mater. Phys., 2010, 81, 184102.

36 SADABS, version 2.05, Bruker AXS, Madison, USA, 1999.

37 M. N. Schneider, T. Rosenthal, C. Stiewe and O. Oeckler, Z. Kristallogr., 2010, 225, 463. 
38 T. Rosenthal, P. Urban, K. Nimmrich, L. Schenk, J. de Boor, C. Stiewe and O. Oeckler, Chem. Mater., 2014, 26, 2567.

39 H. J. Goldsmid and J. W. Sharp, J. Electron. Mater., 1999, 28, 869.

40 A. F. May and G. J. Snyder, in Introduction to Modeling Thermoelectric Transport at High Temperatures in Thermoelectrics and its Energy Harvesting, ed. D. M. Rowe, CRC Press, 2012, vol. 1 .

41 X. Shi, J. Yang, J. R. Salvador, M. Chi, J. Y. Cho, H. Wang, S. Bai, J. Yang, W. Zhang and L. Chen, J. Am. Chem. Soc., 2011, 133, 7837.

42 E. S. Toberer, A. Zevalkink and G. J. Snyder, J. Mater. Chem., 2011, 21, 15843. 